\title{
Sunitinib Rechallenge After Other Targeted Therapies in Metastatic Renal Cell Carcinoma Patients: A Single-Center, Retrospective Study
}

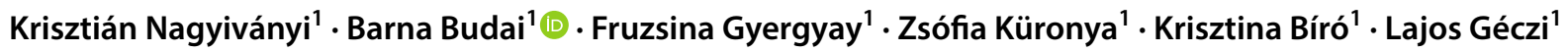

Published online: 26 March 2019

(c) The Author(s) 2019

\begin{abstract}
Background Sunitinib is still one of the standard therapies in metastatic renal cell carcinoma (mRCC). Despite the benefit of sunitinib resistance will develop in the majority of patients. Most of them receive multiple sequential therapies during the course of disease.

Objectives To retrospectively investigate the efficacy and safety of rechallenged sunitinib in third or later line settings.

Patients and Methods Twenty-one mRCC patients were identified who received rechallenged sunitinib between March 2010 and April 2018. Patients received sunitinib in first or second line, then other tyrosine kinase and/or mTOR inhibitors were applied, then sunitinib was rechallenged. Patients' characteristics, tolerability, treatment modalities, and treatment outcomes were recorded. The primary end-point was progression-free survival (PFS) of rechallenged sunitinib.

Results Median age of patients was 62 years at the start of sunitinib rechallenge. Sixty-seven percent of patients were male. All patients had prior nephrectomy. Upon rechallenge 4 patients achieved partial response and 12 stable disease. The median PFS of first sunitinib treatment was 22 (95\% CI 17-26) months and for rechallenged sunitinib 14 (95\% CI 6-20) months. No increased severity of prior toxicity or new adverse events was reported during rechallenged sunitinib. The median overall survival (OS) from the start of first sunitinib was 67 (95\% CI 46-76) months. Multivariate Cox regression analysis revealed that younger age $(<57$ years) at start of first sunitinib ( $\mathrm{HR}=0.24 ; 95 \% \mathrm{CI} 0.07-0.79 ; p=0.019)$ and longer ( $>2$ years) first sunitinib treatment $(\mathrm{HR}=0.28 ; 95 \%$ CI $0.09-0.93 ; p=0.038)$ were independent markers of longer OS.
\end{abstract}

Conclusion Sunitinib rechallenge is a feasible and tolerable option with clinical benefit in selected mRCC patients.

\section{Key Points}

The efficacy of rechallenged sunitinib not depends on the type of targeted treatment(s) applied between the two (first and rechallenged) sunitinib exposures.

There are no differences between the pattern of adverse events related to the first and rechallenged sunitinib treatment.

The overall survival (considered from the start of first sunitinib) depends on the duration of first sunitinib treatment irrespective of consequently applied targeted therapies.

Barna Budai

budai@oncol.hu

1 National Institute of Oncology, Rath Gy. u. 7-9, 1122 Budapest, Hungary

\section{Introduction}

Vascular endothelial growth factor (VEGF) is a key pathway involved in tumor angiogenesis and plays a significant role in the progression of renal cell carcinoma (RCC). A series of randomized clinical trials have shown that molecular targeting of the VEGF receptor pathway improves outcomes in patients with $\mathrm{mRCC}$, in terms of both progression-free survival (PFS) and overall survival (OS), compared with placebo or cytokine therapy [1].

Sunitinib was the first comprehensively studied targeted drug, which is an oral, multi-targeted inhibitor of VEGF receptor 1, 2, and 3, platelet-derived growth factor receptors- $\alpha$ and $-\beta$, and other receptor tyrosine kinases. Sunitinib was approved in Europe in 2006 for the treatment of $\mathrm{mRCC}$ and has since become a reference standard of care for first-line therapy mRCC with good or intermediate Memorial Sloan-Kettering Cancer Center (MSKCC) risk criteria [2]. 
Despite the benefits of sunitinib in terms of PFS and disease stabilization, all patients develop resistance and eventually relapse. The mechanisms of resistance are various and multifactorial, the precise pathway by which a tumor progresses during a given VEGF-targeted therapy is unknown [3].

Treatment guidelines recommend sequential therapy. After disease progression during treatment with one agent, a therapy with another agent usually controls the disease and gives additional clinical benefit. Patients with favorable underlying biology are the most likely to receive several therapies. Switching to mTOR inhibition after a tyrosinekinase inhibitor (TKI) failure in terms of targeting a different signaling pathway resulted in clinical benefit [4]. The current long-term treatment strategy in $\mathrm{mRCC}$ is to give multiple sequential treatments using different agents, there are increasing numbers of studies and case reports suggesting that rechallenge with a specific drug can be of therapeutic benefit [1]. Besides other settings, another TKI or rechallenge with the same TKI is considered as an option according to the recommendations of ESMO clinical practice guideline for patients previously treated with TKI and an mTOR inhibitor [5]. The TKI rechallenge does not fit the present and future mainstream treatment algorithms. According to the recently adopted international recommendation the use of sunitinib in first line was almost pushed out by the new TKI (cabozantinib) and especially by the immunotherapeutic agents (nivolumab + ipilimumab) [6]. The use of TKI rechallenge is suggested for those countries where the introduction of new therapeutic modalities is difficult because of financial reasons.

Here we report the results of a single-center retrospective study. The primary objective was to evaluate the efficacy, as measured by best response, progression-free survival (PFS) and safety of rechallenged sunitinib in $\mathrm{mRCC}$ patients.

\section{Patients and Methods}

\subsection{Patients}

A retrospective study was conducted at the Department of Chemotherapy $\mathrm{C}$ and Clinical Pharmacology of the National Institute of Oncology in Budapest from March 2010 to April 2018. Eligibility included patients with confirmed mRCC (any histology) who previously received sunitinib until progression in first- or second-line, then other TKI and/or mTOR inhibitor was applied, then at the time of progression sunitinib was rechallenged. Data on both sunitinib treatment regimens, intervening therapies were considered. Also, the best response, PFS, side effects and OS also were recorded. Tumor assessment were guided by Response Evaluation Criteria in Solid Tumors (RECIST) version 1.1, treatment-associated adverse events (AEs) were evaluated according to NCI CTCEA v5.0.

\subsection{Statistics}

PFS was calculated from the start of sunitinib treatment until progression according to RECIST. OS was considered as the interval between the start of first sunitinib treatment until death or loss of follow-up. For survival curves, KaplanMeier method was applied. Independent markers of survival, if any, were tested by multivariate Cox regression analysis. A $p$ value of $<0.05$ was considered as statistically significant. For all statistical evaluations the NCSS program [NCSS 12 Statistical Software (2018). NCSS, LLC. Kaysville, Utah, USA] was used.

\section{Results}

The clinicopathologic characteristics of patients are presented in Table 1.

The consecutive treatments of each patient are illustrated in Fig. 1.

Three subgroups were considered according to the treatment type (TKIs: sorafenib $(n=11)$ or axitinib $(n=2)$; mTOR inhibitors: everolimus $(n=12)$ or temsirolimus $(n=1)$; or both), which were administered between sunitinib treatments. It seems that the OS (considered from the start of first sunitinib) depends on the duration of first sunitinib treatment irrespective of systemic therapies applied between sunitinib treatments (Fig. 2). Between sunitinib treatments the mTOR inhibitors $(n=14)$ were applicable for longer than TKIs ( $n=13)$, (mean 9.2 vs 5.7 months/patient); however, this comparison was not the aim of this study.

The best response during first sunitinib treatment (Table 1) was $38 \%$ objective response (OR) and $86 \%$ clinical benefit (CB). The median PFS of first sunitinib treatment was 22 (95\% CI 17-26) months (Fig. 3).

Dose reduction was necessary due to toxicity in one-third of patients. The starting dose of sunitinib was $50 \mathrm{mg}$, except in one patient, who started with $25 \mathrm{mg}$, while the dose was reduced to $37.5 \mathrm{mg}$ in other six patients, and further to $25 \mathrm{mg}$ in two patients.

Multivariate Cox analysis demonstrated that the presence of dose reduction [hazard ratio (HR) $=0.17 ; 95 \%$ CI $0.04-0.78 ; p=0.022$ ] and previous cytokine therapy (HR $=0.29 ; 95 \%$ CI 0.09-0.95; $p=0.042)$ are independent markers of longer PFS for the first sunitinib treatment.

The sunitinib was rechallenged because of progression in all cases. The OR and CB for rechallenged sunitinib was $19 \%$ and $76 \%$, respectively (Table 1). The median PFS of rechallenged sunitinib was 14 (95\% CI 6-20) months 
Table 1 Clinicopathologic characteristics of patients and treatment outcome

\begin{tabular}{|c|c|}
\hline Parameters & Value \\
\hline Age at nephrectomy, years & $49(37-70)$ \\
\hline Age at first sunitinib start, years & $56(37-80)$ \\
\hline Age at sunitinib rechallenge, years & $62(39-83)$ \\
\hline Gender, male & $14(67)$ \\
\hline Histology, clear cell & $19(90)$ \\
\hline MSKCC prognostic index, good & $12(57)$ \\
\hline Metastasis synchronicity, synchronous & $8(38)$ \\
\hline Metastasis localization, multiple & $9(43)$ \\
\hline Lung & $15(71)$ \\
\hline Mediastinal LN & $5(24)$ \\
\hline Adrenal gland & $4(19)$ \\
\hline Liver & $3(14)$ \\
\hline Local recurrence & $3(14)$ \\
\hline Bone & $2(10)$ \\
\hline Other (brain, retroperitoneal LN, skin) & $3(14)$ \\
\hline New metastasis localization during first sunitinib & $7(33)$ \\
\hline New metastasis localization during rechallenged sunitinib & $10(48)$ \\
\hline Cytokine therapy before first sunitinib & $11(52)$ \\
\hline Duration of first sunitinib, months & $22(3-100)$ \\
\hline Dose reduction during first sunitinib & $7(33)$ \\
\hline Best response for first sunitinib, $\mathrm{PR} / \mathrm{SD} / \mathrm{PD}$ & $8 / 10 / 3(38 / 48 / 14)$ \\
\hline Systemic therapy between sunitinib treatments, TKI/mTOR/both & $7 / 8 / 6(33 / 38 / 29)$ \\
\hline Interval between sunitinib treatments, months & $14(3-53)$ \\
\hline Duration of rechallenged sunitinib, months & $10(2-47)$ \\
\hline Dose reduction during rechallenged sunitinib & $3(14)$ \\
\hline Best response for rechallenged sunitinib treatment, $\mathrm{PR} / \mathrm{SD} / \mathrm{PD}$ & $4 / 12 / 5(19 / 57 / 24)$ \\
\hline Systemic treatment after rechallenged sunitinib, TKI/mTOR/both & $2 / 1 / 2(10 / 5 / 10)$ \\
\hline
\end{tabular}

Values are expressed as median (range) or $n(\%)$

$L N$ lymph node, MSKCC Memorial Sloan Kettering Cancer Center, $m T O R$ mTOR pathway inhibitor, $P D$ progressive disease, $P R$ partial response, $S D$ stable disease, $T K I$ tyrosine kinase inhibitor (other than sunitinib)

(Fig. 3). The rechallenged sunitinib was applied in reduced dose $(25 \mathrm{mg})$ for three patients.

No independent markers were found by multivariate Cox analysis of PFS for rechallenged sunitinib.

The toxicity profile of first and rechallenged sunitinib is presented in Table 2.

There were no statistically significant differences between the pattern of AEs related to the first and to the rechallenged sunitinib treatments.

After a median follow-up of 146 months, the median OS from the start of first sunitinib was 67 (95\% CI 46-74) months (Fig. 3). Considering the date of nephrectomy, the median OS proved to be 111 (95\% CI 61-122) months.

Multivariate Cox regression revealed that younger age ( $\leq 56$ years) at start of first sunitinib $(\mathrm{HR}=0.24 ; 95 \% \mathrm{CI}$ $0.07-0.79 ; p=0.019$ ) and longer ( $>2$ years) first sunitinib treatment $(\mathrm{HR}=0.28 ; 95 \%$ CI $0.09-0.93 ; p=0.038)$ were independent markers of longer OS.
At the end of follow-up, three patients were alive, and among them one is still on sunitinib treatment.

\section{Discussion}

The present study is the final evaluation of our previously presented preliminary data [7] investigating sunitinib rechallenge in patients with $\mathrm{mRCC}$. As well as retrospective investigations [8-11] and case reports [12-14], the preliminary result of a recent prospective study was also presented [15] (Table 3). In another study, it was stated that re-exposure to TKI after previous sunitinib and subsequent everolimus treatment may have clinical benefit, but unfortunately the sunitinib rechallenge was not explicitly reported [16]. Other case studies included in some reviews $[8,17]$ were excluded from our list because they were not true rechallenges (patients were not treated with systemic drugs between 
Fig. 1 Treatment schedule of each patient according to drug type(s) applied between sunitinib therapies and duration of first sunitinib treatment. BSC/ $o b s$ best supportive care/observation, $m T O R$ mTOR pathway inhibitor, TKI tyrosine kinase inhibitor (other than sunitinib)

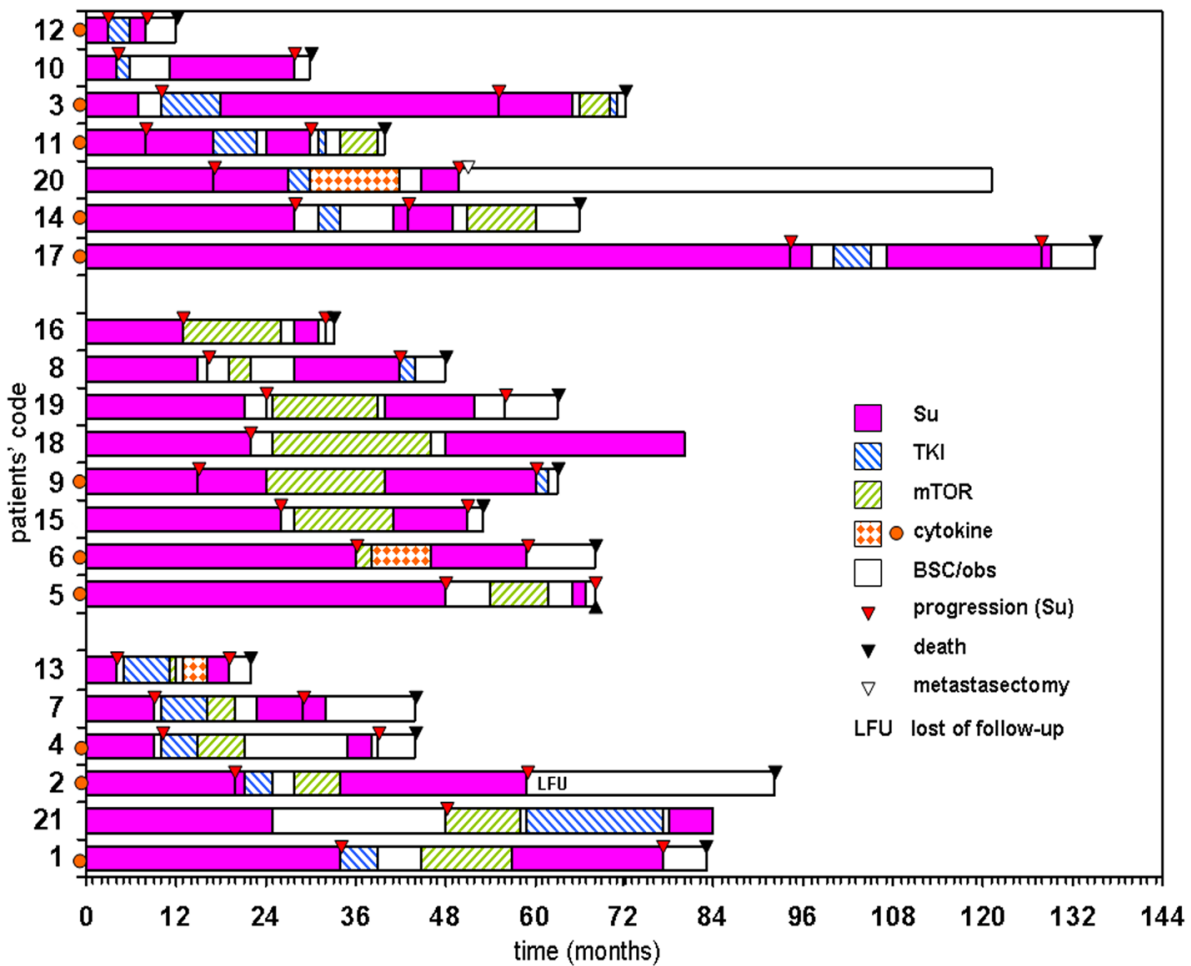

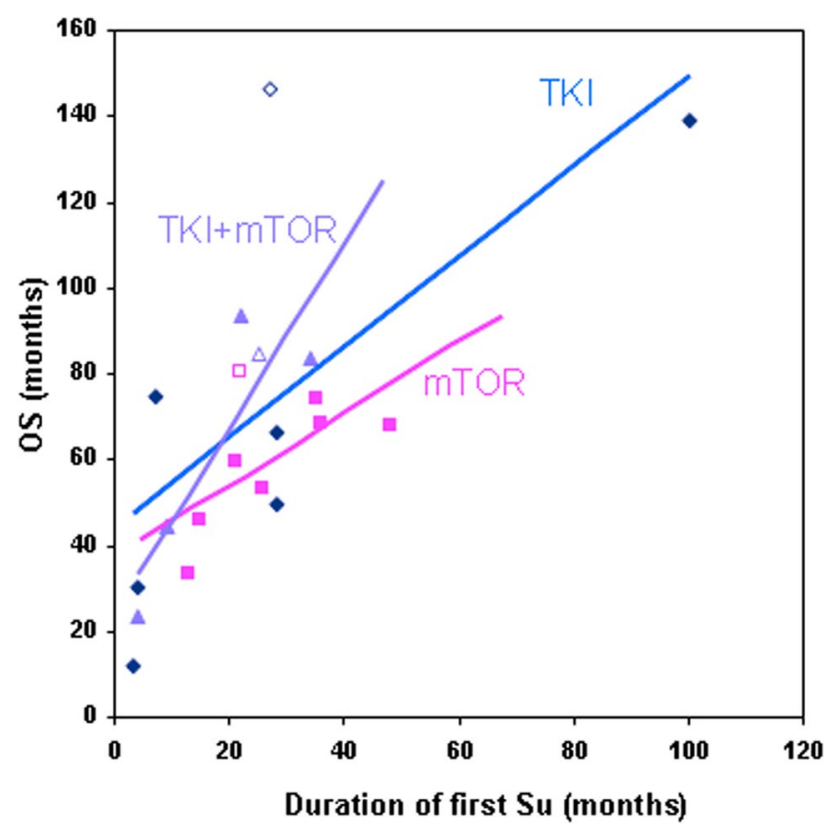

Fig. 2 Correlation between duration of first sunitinib treatment and overall survival (OS). Open marker represents living patients. mTOR mTOR pathway inhibitor, TKI tyrosine kinase inhibitor (other than sunitinib)

sunitinib treatments, therefore they could be considered to have restarted sunitinib [18]).

The above articles all concluded that sunitinib rechallenge is an effective and safe treatment choice for patients who relapsed after sunitinib and subsequent TKIs and/ or mTOR inhibitors. The treatment type between the two sunitinib exposures seemed to be indifferent regarding the efficacy of rechallenged sunitinib; however, only our report and two other authors presented cases when TKIs (other than sunitinib) were applied $[9,10]$. The above statement is strengthened by the above-mentioned prospective study [15] the preliminary results of which were very similar to that of other retrospective studies (Table 3). In that study, sunitinib was applied only for first-line therapy, and everolimus was the second-line therapy for all patients. In contrast, in the other (retrospective) trials, the first sunitinib treatment was applied in different lines (1-4) and between sunitinib treatments different types and number of lines were commenced.

In general, the PFS for rechallenged sunitinib is shorter than that of first sunitinib treatment (Table 3).

The treatments beyond rechallenged sunitinib are not reported, except one case study [13] where another TKI was administered. In our study about one fourth of patients received TKI and/or mTOR after rechallenged sunitinib.

The correlation between duration of first sunitinib and OS, which was demonstrated in our study, had already been described for mRCC treated with sunitinib or cytokine [19]. Also, the longer OS of younger patients observed in our investigation had previously been presented [20]. Further studies regarding these correlations might be warranted.

The conclusion of our previous publication [21] that patients presenting AEs had longer survival, was also 
Fig. 3 Progression-free survival (PFS) of patients after first and rechallenged sunitinib and overall survival (OS) of patients from the start of first sunitinib treatment

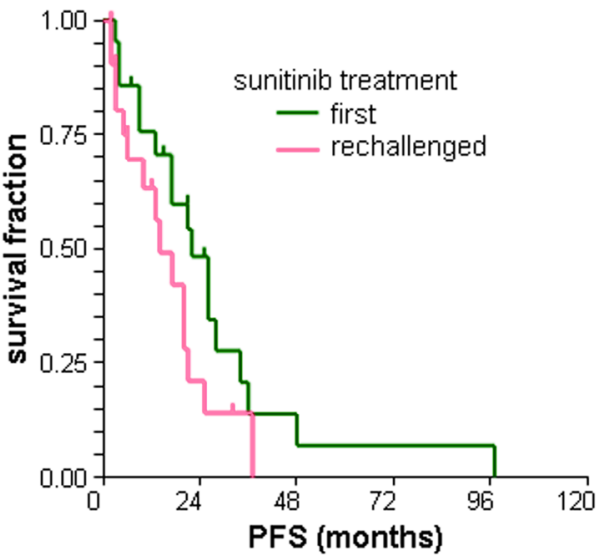

Table 2 Adverse events during first and rechallenged sunitinib treatment

\begin{tabular}{|c|c|c|c|c|}
\hline \multirow[t]{2}{*}{ Adverse events } & \multicolumn{2}{|c|}{ First sunitinib $N(\%)$} & \multicolumn{2}{|c|}{$\begin{array}{l}\text { Rechallenged suni- } \\
\text { tinib } N(\%)\end{array}$} \\
\hline & Grade $1 / 2$ & Grade $3 / 4$ & Grade $1 / 2$ & Grade $3 / 4$ \\
\hline Diarrhea & $12(57)$ & & $13(62)$ & \\
\hline Hypothyreosis & $12(57)$ & & $14(67)$ & \\
\hline Hypertension & $12(57)$ & & $10(48)$ & \\
\hline Anemia & $11(52)$ & & $16(76)$ & \\
\hline Mucositis & $9(43)$ & & $9(43)$ & \\
\hline Thrombocytopenia & $5(24)$ & $3(14)$ & $8(38)$ & $2(10)$ \\
\hline Renal toxicity & $7(33)$ & $1(5)$ & $6(29)$ & $1(5)$ \\
\hline Hand-foot syndrome & $6(29)$ & & $5(24)$ & \\
\hline Vomiting & $5(24)$ & & $3(14)$ & \\
\hline Hepatic toxicity & & $5(24)$ & & $3(14)$ \\
\hline Leucopenia & $3(14)$ & & $8(38)$ & \\
\hline $\begin{array}{l}\text { Cardiovascular toxic- } \\
\text { ity }\end{array}$ & & $1(5)$ & & \\
\hline Other & $5(24)$ & $2(10)$ & $2(10)$ & $1(5)$ \\
\hline
\end{tabular}

observed in this study: dose reduction, a reflection of AEs, resulted in a longer PFS during the first sunitinib treatment.

The longer PFS of patients treated with cytokines before the first sunitinib compared to the first-line sunitinib was an interesting issue and it was clearly demonstrated in a retrospective trial [22]; however, in our study the difference did not reach the level of significance $(p=0.137)$.

Oudard et al. [9] reported significantly longer PFS of rechallenged sunitinib for patients having longer PFS for the first sunitinib. Compared to our study (29\%) they had many more patients (78\%) receiving both mTOR and TKI between sunitinib treatments. Interestingly, the above finding was exclusively observed in case of our six patients (mTOR + TKI), but the difference was not significant $(p=0.114)$, probably due to the low number of patients in this group.

In a study by Zama et al. [10] the interval length $>6$ months between the two sunitinib treatments proved to be a marker of longer PFS of rechallenged sunitinib. This correlation in our study was not checked because

Table 3 Synopsis of published and present results about rechallenged sunitinib

\begin{tabular}{|c|c|c|c|c|c|c|c|c|c|c|}
\hline \multirow{2}{*}{$\begin{array}{l}\text { Study } \\
\text { Reference number }\end{array}$} & \multirow[t]{2}{*}{$n$} & \multirow[t]{2}{*}{ CK [\%] } & \multicolumn{2}{|c|}{ First sunitinib } & \multicolumn{3}{|c|}{ Between } & \multicolumn{2}{|c|}{ Rechallenged sunitinib } & \multirow[t]{2}{*}{$\mathrm{mOS}(\mathrm{mo})$} \\
\hline & & & $\mathrm{OR}(\mathrm{CB})$ & mPFS (mo) & $\begin{array}{l}\text { mTOR } \\
{[\%]}\end{array}$ & $\begin{array}{l}\text { TKI } \\
{[\%]}\end{array}$ & $\begin{array}{l}\text { Both } \\
\text { [\%] }\end{array}$ & $\mathrm{OR}(\mathrm{CB})$ & mPFS (mo) & \\
\hline [9] & 52 & - & $54(94)$ & 18.4 & 15 & 4 & 78 & $15(61)$ & 7.9 & 55.9 \\
\hline$[15]$ & 39 & 0 & $-(100)$ & $>10$ & 100 & 0 & 0 & - & 8.6 & - \\
\hline [10] & 23 & 73 & $65(94)$ & 13.7 & 26 & 35 & 13 & $21(92)$ & 7.2 & - \\
\hline [11] & 13 & 62 & $69(92)$ & 21 & 85 & 0 & 15 & $15(92)$ & 6.9 & NR \\
\hline$[8]$ & 5 & 0 & $-(100)$ & 11.5 & 60 & 0 & 40 & $0(100)$ & 6.4 & - \\
\hline$[12]$ & 1 & 0 & $0(100)$ & 9 & 0 & 0 & 100 & $0(100)$ & $>8$ & $>23$ \\
\hline$[13]$ & 1 & 100 & $0(100)$ & 4 & 100 & 0 & 0 & 100 & 7 & $>38$ \\
\hline [14] & 1 & 0 & 100 & 18 & 100 & 0 & 0 & $100(100)$ & $>7$ & $>30$ \\
\hline Present & 21 & 52 & $38(86)$ & 22 & 38 & 33 & 29 & $19(76)$ & 14 & 67 \\
\hline
\end{tabular}

$C B$ clinical benefit rate, $C K$ cytokine treatment before first sunitinib, italics prospective study, $m o$ months, $m O S$ median overall survival, $m P F S$ median progression-free survival, $m T O R$ mTOR pathway inhibitor, $N R$ not reached, $O R$ objective response rate, TKI tyrosine kinase inhibitor (other than sunitinib) 
only one patient had $<6$ months interval between sunitinib treatments. Considering the median interval (14 months) the difference was non-significant $(p=0.444)$.

Unfortunately, we could not find any predictive marker of PFS of rechallenged sunitinib.

The non-significant difference between the AEs of first and rechallenged sunitinib reported in our study was also clearly demonstrated in detail by Zama et al. [10].

There are several hypotheses for the mechanism, which orchestrate transient nature of sunitinib resistance. The description of these hypotheses goes beyond the scope of this article. Several reviews were already published about sunitinib resistance and rechallenge in RCC [1, 3, 23, 24] and Felicetti et al. [17] summarized the supposed mechanisms for the transient resistance.

Our study has several limitations because of its retrospective nature, the relatively low number of patients and some laboratory parameters were not evaluated. In spite of limitations, this is the first retrospective cohort study of sunitinib rechallenge where the treatment schedules of each patients were presented in detail and relationships between OS and other factors were investigated.

As mentioned in the Introduction section, the new treatment options for mRCC recently adopted as international recommendations have gone beyond sunitinib treatment. Nonetheless, it should be noted that although costs of TKI treatment are high, costs of immunotherapy are many times higher. TKI rechallenge is suggested for those countries where the introduction of new TKI or immunotherapeutic modalities is still delayed because of limited financial resources.

\section{Conclusion}

Sunitinib can be rechallenged after different targeted treatments, and its efficacy does not depends on the type of treatment(s) applied between the first and rechallenged sunitinib exposures. Re-applying sunitinib does not need further caution from oncologists because there were no differences between the pattern of adverse events related to the first and rechallenged sunitinib treatment. The overall survival of patients with $\mathrm{mRCC}$ is markedly influenced by the duration of first sunitinib treatment.

Finally, we fully agree with the statement of Porta et al. [15] that "although many agents are presently available from second line on, in countries where treatment options are still limited, sunitinib rechallenge could still represent a reasonable treatment option" for patients with mRCC.

Further investigation is needed into how the recently approved immunotherapy modifies patients' outcome in sequential use and the rechallenge of sunitinib treatment in mRCC.
Acknowledgements Open access funding provided by National Institute of Oncology (OOI).

\section{Compliance with Ethical Standards}

Funding No source of funding.

Conflict of Interest Krisztián Nagyiványi, Barna Budai, Fruzsina Gyergyay, Zsófia Küronya, Krisztina Bíró and Lajos Géczi declare that they have no conflict of interest.

Ethics Approval All procedures in this study were in accordance with the 1964 Helsinki declaration (and its amendments). The Ethical Committee of the institute and the Hungarian Medical Research Council approved the study.

Informed Consent Not required for this retrospective study.

Open Access This article is distributed under the terms of the Creative Commons Attribution-NonCommercial 4.0 International License (http://creativecommons.org/licenses/by-nc/4.0/), which permits any noncommercial use, distribution, and reproduction in any medium, provided you give appropriate credit to the original author(s) and the source, provide a link to the Creative Commons license, and indicate if changes were made.

\section{References}

1. Oudard S, Vano Y. The role of rechallenge with targeted therapies in metastatic renal-cell carcinoma. Curr Opin Urol. 2015;25:402-10.

2. National Comprehensive Cancer Network. NCCN clinical practice guidelines in oncology-Kidney cancer. https://www.nccn.org/ professionals/physician_gls/pdf/kidney.pdf. Accessed 7 Sep 2018.

3. Morais C. Sunitinib resistance in renal cell carcinoma. J Kidney Cancer VHL. 2014;1:1-11.

4. Rizzo M, Porta C. Sunitinib in the treatment of renal cell carcinoma: an update on recent evidence. Ther Adv Urol. 2017;9:195-207.

5. Escudier B, Porta C, Schmidinger M, Rioux-Leclercq N, Bex A, Khoo V, et al. Renal cell carcinoma: ESMO clinical practice guidelines for diagnosis, treatment and follow-up. Ann Oncol. 2016;27(suppl 5):v58-68.

6. Escudier B, Porta C, Schmidinger M, Rioux-Leclercq N, Bex A, Khoo V, et al. Renal cell carcinoma: ESMO Clinical Practice Guidelines for diagnosis, treatment and follow-up. Ann Oncol. 2019; https://doi.org/10.1093/annonc/mdz056.

7. Nagyiványi K, Bíró K, Gyergyay F, Küronya Z, Németh $H$, Géczi L. Sunitinib rechallenge in metastatic renal cell carcinoma patients. Ann Oncol. 2012;23(Suppl 9):abstract 849P. https://doi. org/10.1093/annonc/mds399.

8. Porta C, Paglino C, Grünwald V. Sunitinib re-challenge in advanced renal-cell carcinoma. Br J Cancer. 2014;111:1047-53.

9. Oudard S, Geoffrois L, Guillot A, Chevreau C, Deville JL, Falkowski S, et al. Clinical activity of sunitinib rechallenge in metastatic renal cell carcinoma-Results of the REchallenge with SUnitinib in MEtastatic RCC (RESUME) Study. Eur J Cancer. 2016;62:28-35.

10. Zama IN, Hutson TE, Elson P, Cleary JM, Choueiri TK, Heng DY, et al. Sunitinib rechallenge in metastatic renal cell carcinoma patients. Cancer. 2010;116:5400-6. 
11. Grünwald V, Weikert S, Seidel C, Busch J, Johannsen A, Fenner M, et al. Efficacy of sunitinib re-exposure after failure of an mTOR inhibitor in patients with metastatic RCC. Onkologie. 2011;34:310-4.

12. Daste A, Gross-Goupil M, Quivy A, François L, Bernhard JC, Ravaud A. Efficacy of rechallenge of metastatic renal cell carcinoma patient with sunitinib after prior resistance to axitinib: case report and review of the literature. Clin Genitourin Cancer. 2016;14:e525-7.

13. Yoshinaga A, Kamata S. Efficacy of sunitinib rechallenge in metastatic renal cell carcinoma: a case report. Hinyokika Kiyo. 2015;61:201-5.

14. Ravaud A, Digue L, Trufflandier N, Smith D. VEGFR TKI 'resistance' or transient clinical insensitivity to VEGFR TKI in metastatic renal cell carcinoma. Ann Oncol. 2010;21:431-2.

15. Porta C, Ferrari VD, Zucali PA, Fornarini G, Bernardo A, Falconeri A, et al. Prospective phase II study of sunitinib rechallenge in metastatic renal cell carcinoma (mRCC): A G.I.O.N. trial. J Clin Oncol. 2017;35(15 suppl):e16081.

16. Maute L, Grünwald V, Weikert S, Kube U, Gauler T, Kahl C, et al. Therapy of mRCC beyond mTOR-inhibition in clinical practice: results of a retrospective analysis. J Cancer Res Clin Oncol. 2014;140:823-7.

17. Felicetti F, Nervo A, Piovesan A, Berardelli R, Marchisio F, Gallo $\mathrm{M}$, et al. Tyrosine kinase inhibitors rechallenge in solid tumors: a review of literature and a case description with lenvatinib in thyroid cancer. Expert Rev Anticancer Ther. 2017;17:1093-8.
18. Nagyiványi K, Budai B, Küronya Z, Gyergyay F, Bíró K, Bodrogi $\mathrm{I}$, et al. Outcome of restarted sunitinib treatment in patients with metastatic renal cell carcinoma: a retrospective trial and combined case reports from literature. Pathol Oncol Res. 2019;25:241-7.

19. Négrier S, Bushmakin AG, Cappelleri JC, Korytowsky B, Sandin $\mathrm{R}$, Charbonneau C, et al. Assessment of progression-free survival as a surrogate end-point for overall survival in patients with metastatic renal cell carcinoma. Eur J Cancer. 2014;50:1766-71.

20. Gonzalez E, Alfaro S, Ramos-Esquivel A, Landaverde DU. Sunitinib effectiveness and safety as first line treatment in metastatic renal cell carcinoma, in the Costa Rican population. J Cancer Metastasis Treat. 2016;2:396-401.

21. Nagyiványi K, Budai B, Bíró K, Gyergyay F, Noszek L, Küronya $\mathrm{Z}$, et al. Synergistic survival: a new phenomenon connected to adverse events of first-line sunitinib treatment in advanced renal cell carcinoma. Clin Genitourin Cancer. 2016;14:314-22.

22. Artaç M, Çoşkun HŞ, Korkmaz L, Koçer M, Turhal NS, Engin H, et al. Using interferon alfa before tyrosine kinase inhibitors may increase survival in patients with metastatic renal cell carcinoma: a Turkish Oncology Group (TOG) study. Clin Genitourin Cancer. 2016;14:e347-53.

23. Ravaud A, Gross-Goupil M. Overcoming resistance to tyrosine kinase inhibitors in renal cell carcinoma. Cancer Treat Rev. 2012;38:996-1003.

24. Schmidinger M. Improving outcomes in metastatic clear cell renal cell carcinoma by sequencing therapy. Am Soc Clin Oncol Educ Book. 2014;2014:e228-38. 